\title{
Posttreatment after Lenvatinib in Patients with Advanced Hepatocellular Carcinoma
}

\author{
Keisuke Koroki $^{a}$ Naoya Kanogawa $^{a}$ Susumu Maruta ${ }^{a, b}$ Sadahisa Ogasawara ${ }^{a, c}$ Yotaro lino ${ }^{d}$ \\ Masamichi Obu ${ }^{d}$ Tomomi Okubo ${ }^{e}$ Norio Itokawa ${ }^{\mathrm{e}}{ }^{\mathrm{f}}$ Takahiro Maeda ${ }^{\mathrm{c}}$ Masanori Inoue ${ }^{\mathrm{c}}$ \\ Yuki Haga $^{g}$ Atsuyoshi Seki $^{\text {h }}$ Shinichiro Okabe ${ }^{i}$ Yoshihiro Koma $^{d}$ Ryosaku Azemoto ${ }^{d}$ \\ Masanori Atsukawa $^{\mathrm{e}, \mathrm{f}}$ Ei Itobayashi ${ }^{\mathrm{b}}$ Kenji Ito ${ }^{g}$ Nobuyuki Sugiurag Hideaki Mizumoto $^{\mathrm{h}}$ \\ Hidemi Unozawa ${ }^{a}$ Terunao Iwanaga ${ }^{a}$ Takafumi Sakuma ${ }^{a}$ Naoto Fujita ${ }^{a}$ Hiroaki Kanzaki $^{a}$ \\ Kazufumi Kobayashi ${ }^{a, b}$ Soichiro Kiyono ${ }^{a}$ Masato Nakamura ${ }^{a}$ Tomoko Saito $^{a}$ \\ Takayuki Kondo $^{a}$ Eiichiro Suzuki ${ }^{a}$ Yoshihiko Ooka $^{a}$ Shingo Nakamoto ${ }^{a}$ Akinobu Tawada ${ }^{a}$ j \\ Tetsuhiro Chiba ${ }^{a}$ Makoto Arai ${ }^{a, j}$ Tatsuo Kandaa,k Hitoshi Maruyamaa,l Jun Kato ${ }^{a}$ \\ Naoya Kato ${ }^{a}$
}

aDepartment of Gastroenterology, Graduate School of Medicine, Chiba University, Chiba, Japan; 'bepartment of Gastroenterology, Asahi General Hospital, Asahi, Japan; 'Translational Research and Development Center, Chiba University Hospital, Chiba, Japan; 'Department of Gastroenterology, Kimitsu Chuo Hospital, Kisarazu, Japan; 'Division of Gastroenterology, Department of Internal Medicine, Nippon Medical School Chiba Hokusoh Hospital, Inzai, Japan; fDivision of Gastroenterology and Hepatology, Department of Internal Medicine, Nippon Medical School, Tokyo, Japan; 9 Department of Gastroenterology, National Hospital Organization Chiba Medical Center, Chiba, Japan; ' Department of Gastroenterology, Funabashi Municipal Medical Center, Funabashi, Japan; 'Department of Gastroenterology, Matsudo City General Hospital, Matsudo, Japan; 'Department of Medical Oncology, Graduate School of Medicine, Chiba University, Chiba, Japan; ${ }^{\mathrm{k} D i v i s i o n}$ of Gastroenterology and Hepatology, Department of Medicine, Nihon University School of Medicine, Tokyo, Japan; 'Department of Gastroenterology, Juntendo University School of Medicine, Tokyo, Japan

\section{Keywords}

Hepatocellular carcinoma - Lenvatinib · Posttreatment

\begin{abstract}
Background: There is no standard posttreatment for patients with advanced hepatocellular carcinoma (HCC) in whom lenvatinib therapy has failed. This study aimed to investigate rates of migration to posttreatment after lenvatinib and to explore candidates for second-line agents in the patients with failed lenvatinib therapy. Methods: We retrospectively collected data on patients with advanced HCC who received
\end{abstract}

karger@karger.com www.karger.com/lic

Karger $\stackrel{\text { ' }}{5}$

GOPEN ACCESS
(C) 2021 The Author(s)

Published by S. Karger AG, Basel

This is an Open Access article licensed under the Creative Commons Attribution-NonCommercial-4.0 International License (CC BY-NC) (http://www.karger.com/Services/OpenAccessLicense), applicable to the online version of the article only. Usage and distribution for commercial purposes requires written permission. lenvatinib as the first-line agent in 7 institutions. Results: Overall survival and progression-free survival (PFS) of 178 patients who received lenvatinib as the first-line agent were 13.3 months (95\% confidence interval [CI], 11.5-15.2) and 6.7 months (95\% Cl, 5.6-7.8), respectively. Sixty-nine of 151 patients $(45.7 \%)$ who discontinued lenvatinib moved on to posttreatment. The migration rates from lenvatinib to the

K. Koroki, N. Kanogawa, and S. Maruta contributed equally to this work.

Clinical trial number: The Chiba University Research Ethics Committee approved this study (No. 2896). 
second-line agent and from the second-line agent to the third-line agent were 41.7 and $44.4 \%$, respectively. Based on multivariate analysis, response to lenvatinib (complete or partial response according to modified RECIST) and discontinuation of lenvatinib due to radiological progression, as well as male were associated with a significantly higher probability of migration to posttreatment after lenvatinib. On the other hand, alpha-fetoprotein levels of $400 \mathrm{ng} / \mathrm{mL}$ or higher was correlated with a significantly lower probability of migration to posttreatment after lenvatinib. Of 63 patients who received second-line systemic therapy, 53 (84.2\%) were administered sorafenib. PFS, objective response rate (ORR), and disease control rate (DCR) for sorafenib treatment were 1.8 months $(95 \% \mathrm{Cl}, 0.6-3.0), 1.8 \%$, and $20.8 \%$, respectively. According to the Cox regression hazard model, Child-Pugh class $B$ significantly contributed to shorter PFS. PFS, ORR, and DCR of 22 patients who received regorafenib after lenvatinib in any lines were 3.2 months (range, 1.5-4.9 months), 13.6\%, and $36.3 \%$, respectively. Similarly, PFS, ORR, and DCR of 17 patients who received regorafenib after lenvatinib in the third-line (after sorafenib) were 3.8 months (range, 1.1-6.5 months), $17.6 \%$, and $41.2 \%$, respectively. Conclusion: Sorafenib may not be a candidate for use as a posttreatment agent after lenvatinib, according to the results of the present study. Regorafenib has the potential to become an appropriate posttreatment agent after lenvatinib.

(C) 2021 The Author(s)

Published by S. Karger AG, Basel

\section{Introduction}

Hepatocellular carcinoma (HCC) is one of the most malignant neoplasms and is the third leading cause of cancer death worldwide $[1,2]$. As of the early 2000s, the development of systemic therapies for advanced HCC was much slower than that for other types of cancer [3]. In 2007, sorafenib, a tyrosine kinase inhibitor (TKI) with an activity against Raf kinase and several receptor tyrosine kinases, including vascular endothelial growth factor receptor 2 (VEGFR2), platelet-derived growth factor receptor (PDGFR), FLT3, Ret, and c-Kit, was associated with significantly longer overall survival (OS) than placebo in 2 global phase III studies $[4,5]$. When sorafenib was positioned as the initial standard systemic therapy in advanced HCC, various molecular target agents (MTAs), mostly TKIs, were under development for patients with advanced HCC [6]. However, several novel compounds had failed in phase III trials compared with sorafenib in the first-line setting or compared with placebo in the second-line setting, and there had been no new agent for advanced HCC for nearly a decade [7-12]. In the late 2010s, after "the era of sorafenib," which was the only single agent for patients with advanced HCC, phase III studies showed that MTAs such as regorafenib, lenvatinib, cabozantinib, and ramucirumab were effective [13-16]. These results have ushered in "the era of multi-MTAs," in which several MTAs can be administered sequentially in real-world practice.

Lenvatinib, an oral TKI that targets VEGFRs 1 through 3 , fibroblast growth factor receptors 1 through 4, PDGFR $\beta$, and the RET and KIT oncogenes, demonstrated a treatment effect on OS by statistical confirmation of noninferiority to sorafenib in patients with advanced HCC in a phase III trial (REFLECT trial) $[14,17]$. At present, lenvatinib is positioned as the standard first-line MTA for treatment of HCC and is being approved by regulatory authorities around the world. Several recent studies have confirmed the effectiveness of lenvatinib for advanced HCC in real-world practice, and it is becoming a mainstay in treatment strategies for advanced HCC [18-20].

The major disadvantage of the use of lenvatinib in clinical practice is the lack of a posttreatment after lenvatinib that has shown clear effectiveness since all phase III trials with published results to date have been designed on the premise that sorafenib is a standard first-line agent in patients with advanced HCC $[13-16,21]$. In "the era of multi-MTAs," when several promising agents are available in real-world practice, sequential treatment with MTAs is becoming a mainstream treatment strategy for advanced HCC, as well as for other cancers. Therefore, the establishment of a second-line treatment after the failure of lenvatinib treatment is an urgent clinical issue to be addressed in systemic therapy in patients with advanced HCC. The aim of this study was to explore candidate drugs that might be appropriate as the second-line treatment after lenvatinib with the use of real-world clinical data.

\section{Patients and Methods}

Patients and Clinical Parameters

The study included patients with advanced HCC who received lenvatinib as a first-line agent in 7 institutions in Japan between March 23, 2018 (the date of lenvatinib approval in Japan), and September 30, 2019. We retrospectively collected clinical parameters described below during the entire clinical course of all patients after lenvatinib was administered. In this analysis, the data were locked on July 31, 2020. The study was approved by the Research Ethics Committee of the Graduate School of Medicine, Chiba University (No. 2896). We had access to information that could identify individual patients during or after the data collection. Patient data were anonymized and deidentified before analysis. 
General treatments with lenvatinib were documented in a previous article [19]. Briefly, the respective standard starting doses of lenvatinib for Child-Pugh A patients weighing $60 \mathrm{~kg}$ or more and less than $60 \mathrm{~kg}$ were 12 and $8 \mathrm{mg}$ orally once per day. The starting dose of lenvatinib for Child-Pugh class B patients was $8 \mathrm{mg}$ orally once per day. Dynamic contrast-enhanced computed tomography or magnetic resonance imaging was performed at baseline and every 1-2 months after starting treatment for evaluation of tumor response. Lenvatinib was continued until the physician determined clear progression of disease on radiological imaging or until the occurrence of adverse events that prevented continuation of treatment. In the current Japanese filed practice, sorafenib, regorafenib, and ramucirumab are available for use after lenvatinib treatment under the national social insurance system. Regorafenib is recommended for use after sorafenib on the basis of the criteria of the RESORCE trial [13] and is currently the agent most commonly administered after sorafenib in Japan, although administration of regorafenib directly after sorafenib is not strictly regulated by the Japanese regulatory authority (Pharmaceuticals and Medical Devices Agency). Ramucirumab is limited to patients with advanced HCC with confirmed alpha-fetoprotein (AFP) levels of $400 \mathrm{ng} / \mathrm{mL}$ or higher [16]. Sorafenib and regorafenib were already available at the time of the approval of lenvatinib in Japan. Ramucirumab was approved on June 18,2019 . In this cohort, each physician decided which treatment to subsequently administer after lenvatinib, including MTAs, resection, local ablation, transarterial chemoembolization (TACE), hepatic arterial infusion chemotherapy (HAIC), radiation, or best supportive care. If local tumor control was assumed to contribute to a better prognosis at the time of lenvatinib failure, we selected the best treatment option from among the resection, local ablation, TACE, HAIC, or radiation. We opted to migrate to other MTAs if patients' liver function and Eastern Cooperative Oncology Group Performance Status (ECOG-PS) were both maintained to the extent that MTAs were tolerated. We recommended the best supportive care if patients had poor liver function or low ECOG-PS at the time of lenvatinib failure. If the selection of posttreatment after lenvatinib was in doubt, the treatment was reviewed and decided at the multidisciplinary team meeting of each institution. A standard starting dosage of sorafenib was $800 \mathrm{mg} /$ day $(400$ mg orally twice daily). Based on the physicians' decisions, a reduced starting dosage of $<800 \mathrm{mg}$ /day was given to some patients in the study. A standard starting dosage was $160 \mathrm{mg} /$ day of regorafenib orally once for 3 weeks, followed by 1 week of no treatment for each cycle. A reduced starting dosage of $<160 \mathrm{mg} /$ day regorafenib was allowed in addition to the reduced dosage of sorafenib.

We retrieved medical records of all patients and collected data on the following clinical parameters: baseline demographic data of lenvatinib, date of radiological progression, cause and date of discontinuation of lenvatinib, baseline demographic data of the second- and third-line agents, date of radiological progression, cause and date of discontinuation of each drug after lenvatinib, and date of death or the last follow-up. Radiological assessments were evaluated according to modified RECIST (mRECIST) [22]. Separately, the Common Terminology Criteria for Adverse Events version 4.0 protocol was used for the assessment of adverse events.

\section{Statistical Analysis}

Kaplan-Meier plots of medians with 95\% confidence intervals (CIs) were used for estimating OS. The censoring date was defined as the date of the last follow-up. Progression-free survival (PFS)
Table 1. Baseline characteristics of 178 patients with HCC treated with lenvatinib

\begin{tabular}{lc}
\hline Demographics/characteristics & $\begin{array}{c}\text { All patients } \\
(n=178)(\%)\end{array}$ \\
\hline Sex, male & $145(81.5)$ \\
Age, $>73$ yr old & $84(47.2)$ \\
HBV positive & $25(14.0)$ \\
HCV positive & $70(39.3)$ \\
Alcohol abuse & $50(28.1)$ \\
Body weight, $<60$ kg & $73(41.0)$ \\
ECOG-PS, $\leq 1$ & $169(94.9)$ \\
Child-Pugh score & \\
$\quad 5$ & $93(52.3)$ \\
$\quad 6$ & $57(32.0)$ \\
$\quad \geq 7$ & $28(15.7)$ \\
Number of intrahepatic lesions, $>7$ & $90(50.6)$ \\
Maximum size of intrahepatic lesions, $>50$ mm & $67(37.6)$ \\
Intrahepatic tumor occupation, $\geq 50 \%$ & $16(9.0)$ \\
MVI & $45(25.3)$ \\
EHM & $64(36.0)$ \\
BCLC stage C & $107(60.1)$ \\
AFP, $>400$ ng/mL & $62(34.8)$ \\
Pretreatment & $142(79.8)$ \\
&
\end{tabular}

$\mathrm{HBV}$, hepatitis B virus; HCV, hepatitis C virus; ECOG, Eastern Cooperative Oncology Group; PS, performance status; MVI, macrovascular invasion; EHM, extrahepatic metastasis; BCLC, Barcelona clinic liver cancer; AFP, alpha-fetoprotein; HCC, hepatocellular carcinoma; ECOG-PS, Eastern Cooperative Oncology Group Performance Status.

after lenvatinib and each second- or third-line agent was estimated using Kaplan-Meier plots of medians with 95\% CIs, with the date of progression defined according to mRECIST and the censoring date defined as the date of last radiological assessment without progression. Logistic regression analysis was performed to assess the factors for migration to posttreatment after lenvatinib. The Cox proportional hazard regression model was used to estimate hazard ratios for PFS with sorafenib treatment after lenvatinib. Both multivariate logistical and Cox proportional hazard regressions were calculated by using a stepwise model. A $p$ value of $<0.05$ was considered statistically significant. All statistical analyses were conducted using the Statistical Package for the Social Sciences version 25 statistical software (SPSS, IBM, Armonk, NY, USA).

\section{Results}

\section{Study Population}

During the study period, 178 patients with advanced HCC received lenvatinib as first-line systemic therapy at 7 Japanese institutions. Table 1 shows the baseline characteristics of the study population. The majority of patients $(81.5 \%)$ were male, and the median age was 73 years (range, 

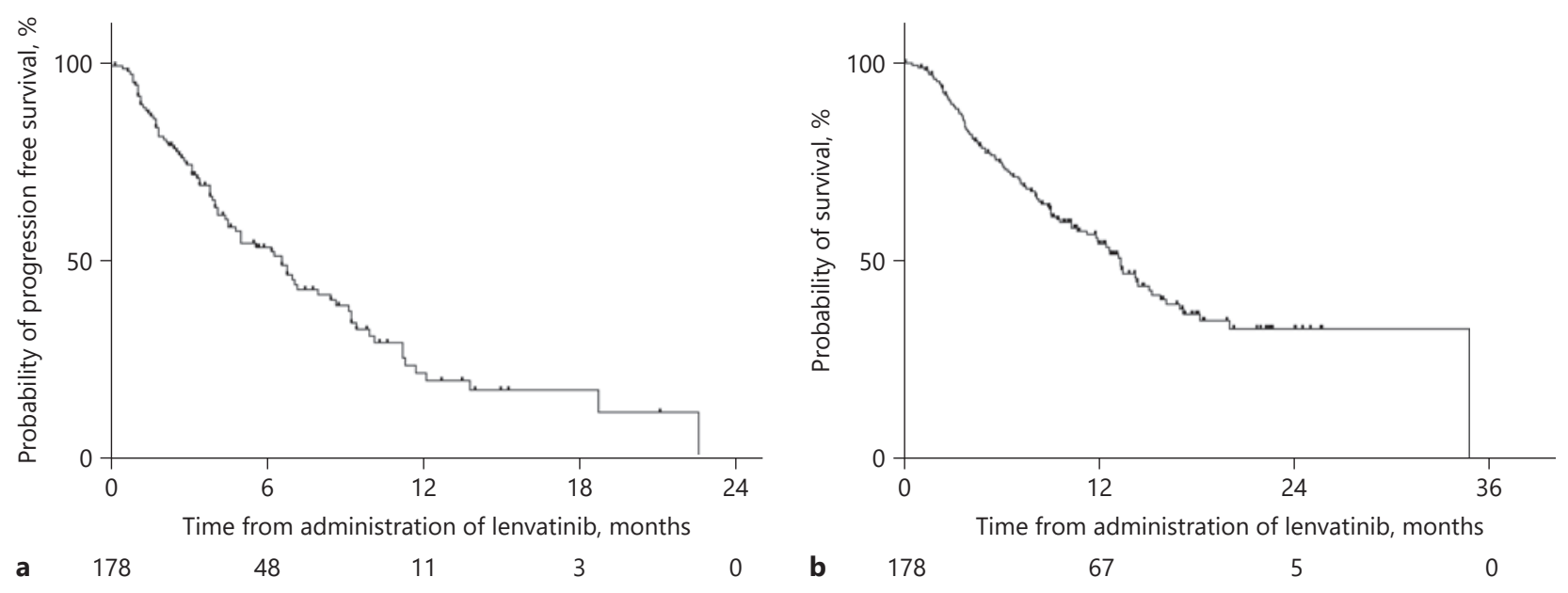

67

5

0

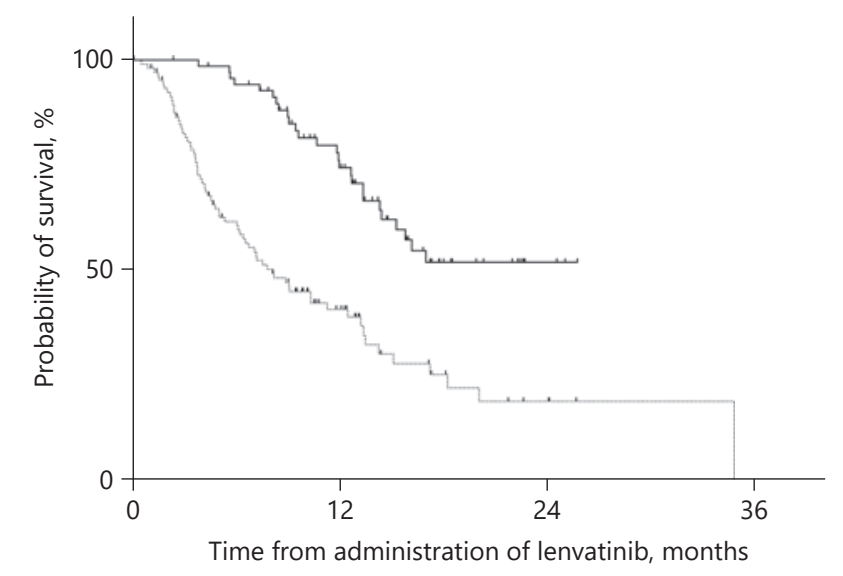

$\begin{array}{lllll}\text { Absent } & 82 & 12 & 1 & 0 \\ \text { Present } & 69 & 42 & 3 & 0 \\ \text { C } & & & \end{array}$

Fig. 1. a PFS with lenvatinib treatment. b OS from administration of lenvatinib. c Comparison of OS between patients without (dotted line) and with (solid line) posttreatment after lenvatinib. PFS, progression-free survival; OS, overall survival.

33-95 years). At the time of lenvatinib administration, most of the patients were ECOG-PS 0 or 1 (94.9\%) and Child-Pugh class A (84.3\%). According to the baseline radiological assessments, 25.3 and $36.0 \%$ of patients had macrovascular invasion and extrahepatic metastasis, respectively. OS and PFS were 13.3 months (95\% CI, 11.5-15.2) and 6.7 months (95\% CI, 5.6-7.8), respectively (Fig. 1). The rate of objective response according to mRECIST was $39.3 \%$. During the follow-up period, 151 of 178 patients (84.8\%) discontinued lenvatinib, and 87 of them (57.2\%) had confirmed radiological progression according to $\mathrm{mRE}$ CIST. The median treatment duration of lenvatinib was 4.9 months (95\% CI, 3.9-5.9). The most common causes of discontinuation of lenvatinib in patients without radiological progression were anorexia (12 patients, $7.9 \%$ ), disease progression determined clinically (without radiological progression according to mRECIST) (12 patients, $7.9 \%$ ), disorder of physical status (10 patients, $6.6 \%)$, fatigue ( 8 patients, $5.3 \%$ ), and liver dysfunction (7 patients, $4.6 \%$ ).

\section{Migration to Posttreatment after Discontinuation of Lenvatinib}

Up to the date of data cutoff, 69 of 151 patients (45.7\%) who discontinued lenvatinib migrated to posttreatment (Fig. 2). The other 82 patients (54.3\%) received palliative care after discontinuation of lenvatinib. The majority of 
Fig. 2. Overview of transition from lenvatinib to second- and third-line treatments in patients with advanced HCC. BSC, best supportive care; MTA, molecular target agent; HCC, hepatocellular carcinoma.

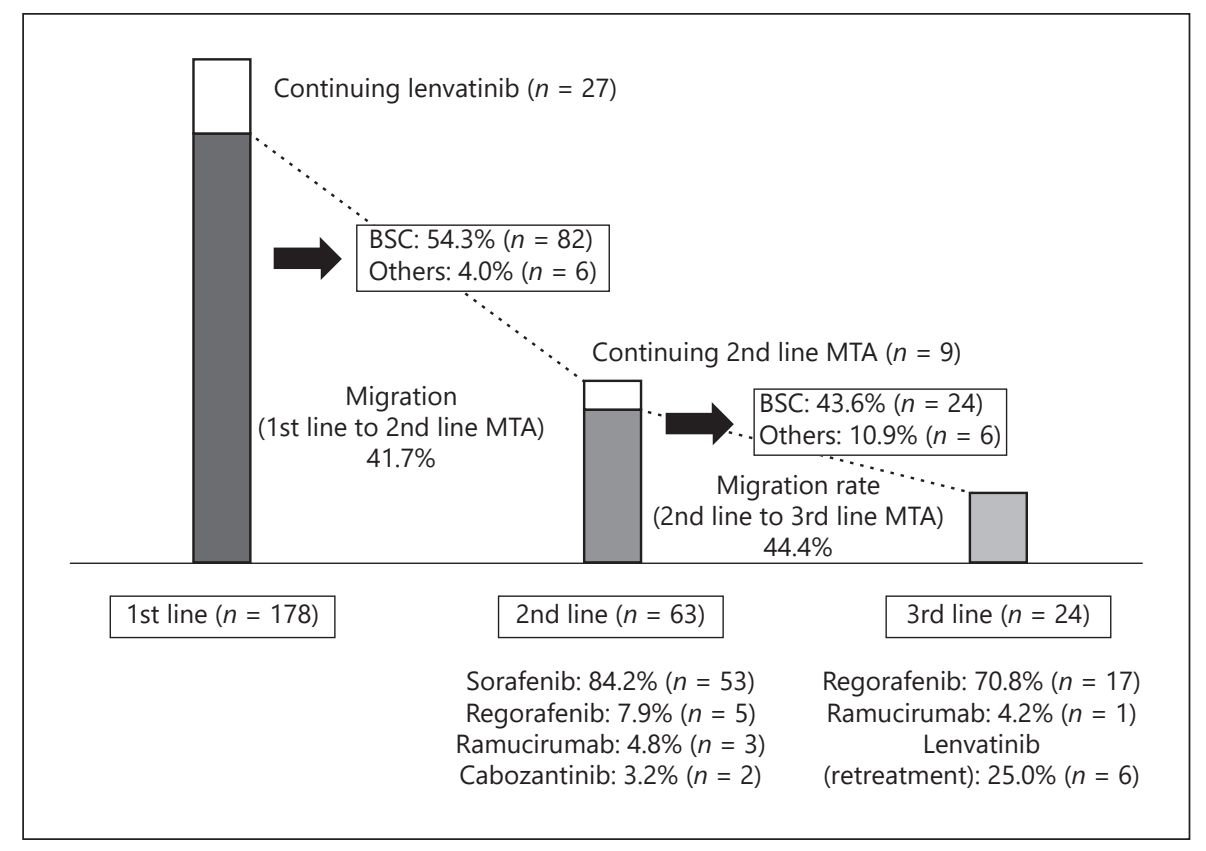

Table 2. Logistic regression analysis of the predictive factors for migration to posttreatment after lenvatinib in patients with advanced HCC

\begin{tabular}{|c|c|c|c|c|c|c|}
\hline Variables & \multicolumn{2}{|c|}{ Univariate analysis } & $p$ value & \multicolumn{2}{|c|}{ Multivariate analysis } & $p$ value \\
\hline Age, $>73$ yr old & 0.626 & $0.328-1.194$ & 0.155 & & & \\
\hline Body weight, $<60 \mathrm{~kg}$ & 0.568 & $0.295-1.093$ & 0.090 & & & \\
\hline ECOG-PS >1 & 0.460 & $0.086-2.447$ & 0.362 & & & \\
\hline HCV positive & 0.821 & $0.428-1.575$ & 0.554 & & & \\
\hline Intrahepatic tumor volume, $>50 \%$ & 1.230 & $0.409-3.697$ & 0.713 & & & \\
\hline BCLC stage $\mathrm{C}$ & 0.426 & $0.218-0.833$ & 0.013 & & & \\
\hline Baseline AFP, $>400 \mathrm{ng} / \mathrm{mL}$ & 0.333 & $0.164-0.675$ & 0.002 & 0.368 & $0.160-0.756$ & 0.008 \\
\hline Responder to lenvatinib & 2.638 & $1.320-5.70$ & 0.006 & 3.081 & $1.387-6.843$ & 0.006 \\
\hline Discontinued lenvatinib after radiological progression & 3.172 & $1.621-6.210$ & 0.001 & 2.260 & $1.087-4.699$ & 0.029 \\
\hline
\end{tabular}

HCC, hepatocellular carcinoma; BCLC, Barcelona clinic liver cancer; ECOG, Eastern Cooperative Oncology Group; HBV, hepatitis B virus; HCV, hepatitis $C$ virus; PS, performance status; AFP, alpha-fetoprotein; CI, confidence interval.

patients who migrated to posttreatment after lenvatinib were given MTAs (63 of 69 patients), but 6 patients received other treatments (TACE, 3 patients; HAIC, 2 patients; and resection, 1 patient). None of the patients in the present cohort had been treated by radiation therapy after lenvatinib failure. Similarly, 54 of 63 patients (85.7\%) who migrated to the second-line agents discontinued treatment, and 30 of them (55.5\%) received posttreatment after the second-line agents (third-line agents, 24 patients; HAIC, 4 patients; unknown, 2 patients). The rates of migration from lenvatinib to the second-line MTAs and from the second-line MTAs to the third-line MTAs were 41.4 and $44.4 \%$, respectively. Of 144 patients with both Child-Pugh A and EOCG-PS scores $\leq 1$ at the time of starting lenvatinib, 123 patients discontinued lenvatinib at the time of data cutoff and 54 patients migrated from lenva- 
Table 3. Transition of liver function between the start and end of lenvatinib according to Child-Pugh score

\begin{tabular}{cccc}
\hline \multirow{2}{*}{ Child-Pugh score } & \multicolumn{3}{c}{ Migration to posttreatment after lenvatinib } \\
\cline { 2 - 4 } & absent (\%) & present (\%) & p value \\
\hline At the time of start of lenvatinib & & \\
5 & $35(43.2)$ & $40(58.8)$ & 0.155 \\
6 & $31(38.3)$ & $20(29.4)$ & \\
$\geq 7$ & $15(18.5)$ & $8(11.8)$ & \\
At the time of end of lenvatinib & & \\
5 & $12(14.8)$ & $24(35.3)$ & $<0.001$ \\
6 & $15(18.5)$ & $20(29.4)$ & \\
$\geq 7$ & $54(66.7)$ & $24(35.3)$ & \\
Transition of liver function & & \\
Improvement & $4(4.9)$ & $5(7.4)$ & \\
Remaining & $20(24.7)$ & $34(50.0)$ & \\
Worsening & $57(70.4)$ & $29(42.6)$ & \\
\end{tabular}

tinib to the second-line MTAs (43.9\%). Furthermore, 23 of 47 patients, excluding 7 patients who were ongoing the second-line MTAs, migrated from the second-line MTAs to the third-line MTAs (48.9\%). Fig. 1c shows that OS after lenvatinib was significantly longer in patients who migrated to posttreatment than in patients who received palliative care (posttreatment, OS not reached; palliative care, OS 6.0 months; 95\% CI, 4.3-7.8; $p<0.001$ ).

We used logistical regression analysis to identify the factors that contributed to migration to posttreatment after lenvatinib (Table 2). In this analysis, patients who received not only MTAs but also other treatments (i.e., TACE, HAIC, and resection) were included. Baseline clinical parameters at the time of lenvatinib administration and clinical outcomes during lenvatinib treatment were included in this analysis. We found that male, responder of lenvatinib, and discontinuation of lenvatinib due to radiological progression according to mRECIST were associated with a significantly higher probability of migration to posttreatment after lenvatinib. On the other hand, AFP level $>400 \mathrm{ng} / \mathrm{mL}$ at the time of lenvatinib administration was a significant negative predictive factor for migration to posttreatment after lenvatinib.

In the present cohort, 149 patients (98.7\%) had the liver function assessed at the time of lenvatinib discontinuation. Table 3 shows transition of liver function between the start and the end of lenvatinib according to ChildPugh scores. Patients who migrated to posttreatment had significantly better Child-Pugh scores at the end of lenvatinib treatment than patients moved to the best supportive care.

\section{Sorafenib after Discontinuation of Lenvatinib}

Of 63 patients who received second-line systemic therapy after failure of lenvatinib treatment, 53 patients $(84.2 \%)$ received sorafenib. The baseline characteristics of patients at the time of sorafenib administration are shown in Table 4. All 53 patients were ECOG-PS 0 or 1, and 36 patients (67.9\%) were Child-Pugh class A. At baseline radiological assessment, $30.2 \%$ of patients had macrovascular invasion and $39.6 \%$ had extrahepatic metastasis. OS and PFS from the date of sorafenib administration were 10.4 months (95\% CI, 4.7-16.0) and 1.8 months (95\% CI, 0.6-3.0), respectively (Fig. 3a). According to mRECIST, the objective response rate (ORR) and the disease control rate (DCR) were 1.8 and $20.8 \%$, respectively. Table 5 shows the results of multivariate analysis of PFS in sorafenib-treated patients in whom lenvatinib treatment failed using the Cox proportional hazard regression model. According to this analysis, Child-Pugh class B significantly contributed to shorter PFS. The most common adverse events during sorafenib treatment were increased aspartate aminotransferase (AST) (58.5\%), hypertension (49.1\%), hypoalbuminemia (47.2\%), proteinuria $(37.7 \%)$, and fatigue (30.2\%) (Table 6). The most common grade 3 or higher adverse events were increased AST (11.3\%) and proteinuria (11.3\%). Up to the date of data cutoff, 48 patients discontinued sorafenib treatment, of which 26 had confirmed radiological progression before discontinuing sorafenib. The most common causes of sorafenib discontinuation in patients without radiological progression were disorder of the physical status (5 patients, 10.4\%), clinically determined disease progression which was assessed by tumor makers ( 5 patients, $10.4 \%$ ), and proteinuria (3 patients, $6.3 \%$ ). Of 35 patients who discontinued lenvatinib because of radiological progression, 20 patients discontinued sorafenib because of radiological progression and 4 patients discontinued sorafenib because of adverse events (proteinuria: 2 patients, disorder of physical status: 2 patients). Of the remaining 18 patients without radiological progression in whom lenvatinib treatment failed, 9 patients discontinued lenvatinib because of clinically determined disease progression and adverse events, respectively. Of the 9 patients who migrated to sorafenib after discontinuation of lenvatinib because of adverse events, 4 patients continued sorafenib until radiological progression. Reasons for discontinuing lenvatinib were increased AST, anorexia, gastrointestinal bleeding, and proteinuria. In the other 5 patients who discontinued both lenvatinib and sorafenib because of adverse events, the causes for lenvatinib discontinuation were fatigue ( 2 patients), rash, proteinuria, and stomati- 
Table 4. Demographics and characteristics of lenvatinib-treated patients with advanced HCC who received sorafenib or regorafenib

\begin{tabular}{|c|c|c|c|}
\hline Demographics/characteristics & $\begin{array}{l}\text { Sorafenib } \\
(n=53)(\%)\end{array}$ & $\begin{array}{l}\text { Regorafenib } \\
\text { (any lines) } \\
(n=22)(\%)\end{array}$ & $\begin{array}{l}\text { Regorafenib } \\
\text { (third line) } \\
(n=17)(\%)\end{array}$ \\
\hline Sex, male & $48(90.6)$ & $19(86.4)$ & $15(88.2)$ \\
\hline Age, $>73$ yr old & $23(43.4)$ & $7(31.8)$ & $5(29.4)$ \\
\hline HBV positive & $6(11.3)$ & $4(18.2)$ & $3(17.6)$ \\
\hline $\mathrm{HCV}$ positive & $23(43.4)$ & $10(45.5)$ & $8(47.1)$ \\
\hline Alcohol abuse & $15(28.3)$ & $4(18.2)$ & $2(11.8)$ \\
\hline Body weight, <60 kg & $30(56.6)$ & $13(59.1)$ & $10(58.8)$ \\
\hline ECOG-PS, $\leq 1$ & $53(100.0)$ & $22(100.0)$ & $17(100.0)$ \\
\hline \multicolumn{4}{|l|}{ Child-Pugh score } \\
\hline 5 & $16(30.2)$ & $8(36.4)$ & $6(35.3)$ \\
\hline 6 & $20(37.7)$ & $9(40.9)$ & $6(35.3)$ \\
\hline$\geq 7$ & $17(32.1)$ & $5(22.7)$ & $5(29.4)$ \\
\hline Number of intrahepatic lesions, $>7$ & $32(60.4)$ & $15(68.2)$ & $12(70.6)$ \\
\hline Maximum size of intrahepatic lesions, $>50 \mathrm{~mm}$ & $23(43.4)$ & $11(50.0)$ & $9(52.9)$ \\
\hline Intrahepatic tumor occupation, $\geq 50 \%$ & $4(7.5)$ & $3(13.6)$ & $3(17.6)$ \\
\hline MVI & $16(30.2)$ & $6(27.3)$ & $5(29.4)$ \\
\hline EHM & $21(39.6)$ & $12(54.5)$ & $10(58.8)$ \\
\hline BCLC stage C & $36(67.9)$ & $15(68.2)$ & $12(70.6)$ \\
\hline $\mathrm{AFP},>400 \mathrm{ng} / \mathrm{mL}$ & $23(43.4)$ & $7(31.8)$ & $4(23.5)$ \\
\hline \multicolumn{4}{|l|}{ Causes for discontinuation of lenvatinib } \\
\hline Radiological progression & $39(73.6)$ & $19(86.1)$ & $14(82.4)$ \\
\hline Adverse events & $10(18.9)$ & $2(9.1)$ & $2(11.8)$ \\
\hline Others & $4(7.5)$ & $1(4.5)$ & $1(5.9)$ \\
\hline \multicolumn{4}{|l|}{ Treatment line } \\
\hline 2nd-line & $53(100.0)$ & $5(22.7)$ & 0 \\
\hline 3rd-line & 0 & $17(77.3)$ & $17(100.0)$ \\
\hline
\end{tabular}

$\mathrm{HBV}$, hepatitis B virus; HCV, hepatitis C virus; ECOG, Eastern Cooperative Oncology Group; PS, performance status; MVI, macrovascular invasion; EHM, extrahepatic metastasis; BCLC, Barcelona clinic liver cancer; AFP, alpha-fetoprotein; HCC, hepatocellular carcinoma; ECOG-PS, Eastern Cooperative Oncology Group Performance Status.

tis. Two of these patients discontinued sorafenib after experiencing the same adverse events as with lenvatinib (fatigue: 1 patient, rash: 1 patient).

\section{Regorafenib after Discontinuation of Lenvatinib}

Twenty-two patients received regorafenib after discontinuation of lenvatinib, as second-line therapy in 5 patients and as third-line therapy in 17 patients. The baseline characteristics at the time of regorafenib administration of all 22 patients who received regorafenib in any lines and 17 patients who received regorafenib after sorafenib (third-line) are shown in Table 4. The median PFS of patients receiving regorafenib in any lines and as third-line therapy was 3.2 months (95\% CI, 1.5-4.9 months) and 3.8 months (95\% CI, 1.1-6.5 months), respectively (Fig. 3b). The ORR and the DCR according to
mRECIST were 13.6 and $36.3 \%$ for therapy in any lines and 17.6 and $41.2 \%$ in third-line therapy, respectively. Among adverse events during regorafenib treatment, we observed high frequencies of increased AST (59.1\%), proteinuria $(54.6 \%)$, hypertension $(45.6 \%)$, fatigue (40.9\%), palmar-plantar erythrodysesthesia (36.4\%), and hypoalbuminemia (36.4\%) (Table 7). Up to the time of data cutoff, 18 patients discontinued regorafenib, of which 8 patients migrated to posttreatment (TACE, 4 patients; ramucirumab, 3 patients; other, 1 patient). Radiological progression was confirmed in 9 patients, and 9 patients discontinued regorafenib due to adverse events. The most common causes of discontinuation of regorafenib were liver dysfunction (2 patients), anorexia (2 patients), and clinically determined disease progression (2 patients). 
Table 5. Cox regression analysis of the factors for PFS with sorafenib treatment after lenvatinib in patients with advanced HCC

\begin{tabular}{|c|c|c|c|c|c|c|}
\hline Variables & hazard ratio & $95 \% \mathrm{CI}$ & $p$ value & hazard ratio & $95 \% \mathrm{CI}$ & $p$ value \\
\hline Age, $>73$ yr old & 0.975 & $0.452-2.103$ & 0.949 & & & \\
\hline Child-Pugh class B & 2.755 & $1.248-6.078$ & 0.012 & 2.755 & $1.248-6.078$ & 0.012 \\
\hline HBV positive & 0.992 & $0.233-4.225$ & 0.992 & & & \\
\hline BCLC C & 1.145 & $0.509-2.577$ & 0.743 & & & \\
\hline Baseline AFP, $>400 \mathrm{ng} / \mathrm{mL}$ & 2.208 & $0.974-5.010$ & 0.058 & & & \\
\hline Responder to lenvatinib & 0.896 & $0.414-1.941$ & 0.781 & & & \\
\hline Discontinued lenvatinib after radiological progression & 1.819 & $0.758-4.367$ & 0.181 & & & \\
\hline Treatment duration of lenvatinib, $>4.9 \mathrm{mo}$ & 1.292 & $0.558-2.988$ & 0.550 & & & \\
\hline
\end{tabular}

HBV, hepatitis B virus; HCV, hepatitis C virus; BCLC, Barcelona clinic liver cancer; HCC, hepatocellular carcinoma; AFP, alphafetoprotein; CI, confidence interval; PFS, progression-free survival.

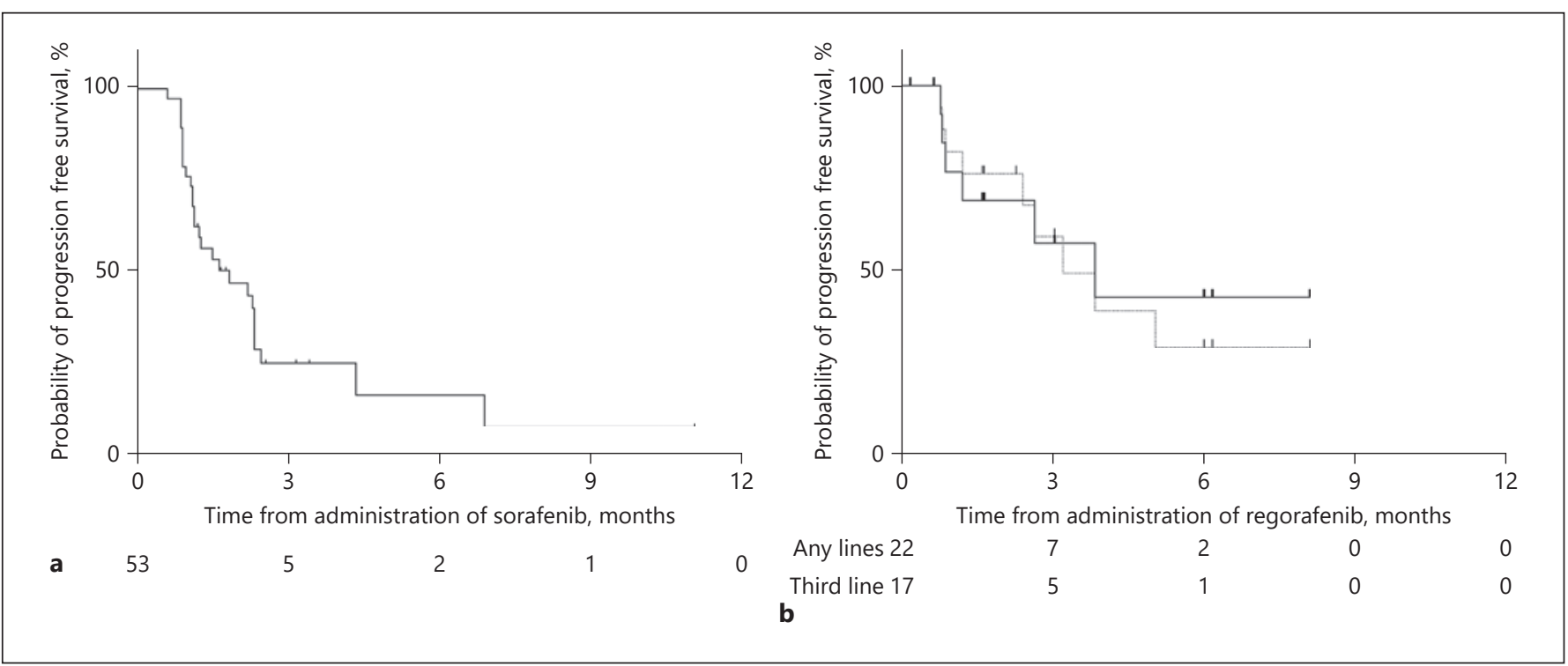

Fig. 3. a PFS in patients receiving sorafenib after lenvatinib. b PFS in patients receiving regorafenib after lenvatinib in any lines (dotted line) and 3rd line (solid line). PFS, progression-free survival.

\section{Discussion}

The present study described the clinical outcomes of patients with advanced HCC who were treated with sorafenib or regorafenib after failure of lenvatinib treatment. PFS, ORR, and DCR in patients treated with sorafenib after lenvatinib were far from what would be expected in patients with advanced HCC treated with MTAs in current clinical practice. On the other hand, the effectiveness of regorafenib after lenvatinib indicated that regorafenib could be a potential second-line agent after lenvatinib.

To the best of our knowledge, this study is the first to report rates of migration to posttreatments and postsystemic therapies after lenvatinib in patients with advanced HCC in real-world practice. According to the post hoc analysis from the phase III study (REFLECT study), $32.6 \%$ of patients with lenvatinib as a first-line agent received any anticancer medication during the follow-up period [23]. 
Table 6. Adverse events (>10\%) during treatment with sorafenib after lenvatinib in patients with advanced HCC

\begin{tabular}{lll}
\hline \multirow{2}{*}{ Events } & \multicolumn{2}{l}{ Sorafenib $(n=53)$} \\
\cline { 2 - 3 } & any $(\%)$ & grade $\geq 3(\%)$ \\
\hline Aspartate aminotransferase increased & $31(58.5)$ & $6(11.3)$ \\
Hypertension & $26(49.1)$ & $1(1.9)$ \\
Hypoalbuminemia & $25(47.2)$ & 0 \\
Proteinuria & $20(37.7)$ & $6(11.3)$ \\
Fatigue & $16(30.2)$ & 0 \\
Thrombocytopenia & $15(28.3)$ & $1(1.9)$ \\
Bilirubin increased & $14(26.4)$ & $2(3.8)$ \\
Hemolysis & $14(26.4)$ & $1(1.9)$ \\
Palmar-plantar erythrodysesthesia & $13(24.5)$ & $1(1.9)$ \\
Anorexia & $13(24.5)$ & 0 \\
Serum amylase increased & $9(17.0)$ & $2(3.8)$ \\
Diarrhea & $7(13.2)$ & $1(1.9)$ \\
Erythema multiform & $6(11.3)$ & $2(3.8)$ \\
Alopecia & $4(7.5)$ & 0 \\
Hypothyroidism & $4(7.5)$ & 0 \\
White blood cell count decreased & $4(7.5)$ & $1(1.9)$ \\
Encephalopathy & $3(5.7)$ & 0 \\
\hline
\end{tabular}

HCC, hepatocellular carcinoma.

Since that study was conducted at a time when second-line MTAs, such as regorafenib, cabozantinib, and ramucirumab were not approved in most countries, the migration rate was lower than those in our study. Hiraoka et al. reported that $43.8 \%$ of patients with confirmed radiological progression during lenvatinib treatment were eligible for postprogression treatment after lenvatinib [24]. This rate of eligibility for postprogression treatment was similar with our rates of migration to posttreatment in realworld practice in patients with advanced HCC who received lenvatinib as a the first-line agent, although Hiraoka et al. excluded Child-Pugh B patients and patients with ECOG-PS $>1$ at the time of lenvatinib administration and included patients who had received previous systemic therapies. Our results also indicated that patients who were able to move on to posttreatment after lenvatinib had better liver function than those who did not migrate to posttreament (Table 3 ). We also found 4 independent factors that contributed to migration to posttreatment after lenvatinib (male, baseline AFP $>400 \mathrm{ng} / \mathrm{mL}$, responder to lenvatinib, and discontinued lenvatinib after radiological progression). Interestingly, none of the 4 factors were related to liver function deterioration individually at the time of lenvatinib failure (online suppl. Supplementary Table; see www.karger.com/doi/10.1159/000515552 for all online suppl. material). These results might suggest that the 4 independent factors contributed to liver function deterioration at the time of lenvatinib failure in a complex manner. Of these 4 factors, the only 1 we can improve in clinical practice is to reduce discontinuation of lenvatinib because of adverse events and continue using it until radiological progression. However, only about half of our patients were able to continue lenvatinib until radiological progression. This rate is similar with that in the report of Hiraoka et al. [24]. Recently, physicians' skills at managing adverse events from sorafenib were reported to improve over time [25]. Both our study and that of Hiraoka et al. were based on patient populations immediately after the approval of lenvatinib in Japan. As physicians improve their understanding of the characteristics of lenvatinib from reports based on real-world practice [18-20, 26-28], their skills at managing adverse events from lenvatinib as well as sorafenib will improve, and the probability of continuing lenvatinib until radiological progression will increase. Moreover, deterioration of liver function and worsening physical condition (so-called performance status) at the time of previous treatment failure are $2 \mathrm{ma}$ jor factors that prohibit patients with advanced HCC from transitioning to other systemic therapy. Skillful managements of adverse events will lead to maintaining both the liver function and physical status until the end of lenvatinib treatment. Increasing the probability of continuing lenvatinib until radiological progression will lead to an increase in the rates of migration to posttreatment after lenvatinib in patients with advanced HCC.

We showed that patients who moved on to posttreatment after lenvatinib had a better prognosis than patients who received palliative care. The analysis from the REFLECT trial showed that OS of patients who received posttreatment after lenvatinib was 20.8 months [23]. Moreover, lenvatinib responders who received any subsequent anticancer medication had a median OS of 25.7 months [23]. Our study found that lenvatinib responders had a significantly high probability of migration to posttreatment after failure of lenvatinib treatment compared with nonresponders. The results of the REFLECT trial and our study suggest that survival may be prolonged by migrating to appropriate postsystemic therapy in responders to lenvatinib who are considered to have a good prognosis [29]. To maximize the potential of lenvatinib in patients with advanced HCC, we should hasten the development of treatment streams after failure of lenvatinib.

The present study found that PFS, ORR, and DCR of patients treated with sorafenib after lenvatinib were 1.8 months, $1.8 \%$, and $20.8 \%$, respectively. These clinical outcomes were even worse than those found in phase III 
Table 7. Adverse events $(>10 \%)$ during treatment with regorafenib after lenvatinib in patients with advanced HCC

\begin{tabular}{|c|c|c|c|c|}
\hline \multirow[t]{2}{*}{ Events } & \multicolumn{2}{|c|}{ Regorafenib (any lines) $(n=22)$} & \multicolumn{2}{|c|}{ Regorafenib (third line) $(n=17)$} \\
\hline & any $(\%)$ & grade $\geq 3(\%)$ & any $(\%)$ & grade $\geq 3(\%)$ \\
\hline Aspartate aminotransferase increased & $13(59.1)$ & $2(9.1)$ & $11(64.7)$ & $2(11.8)$ \\
\hline Proteinuria & $12(54.6)$ & 0 & $9(52.9)$ & 0 \\
\hline Hypertension & $10(45.6)$ & $2(9.1)$ & $9(52.9)$ & $2(11.8)$ \\
\hline Fatigue & $9(40.9)$ & 0 & $7(41.2)$ & 0 \\
\hline Palmar-plantar erythrodysesthesia & $8(36.4)$ & $2(9.1)$ & $4(23.5)$ & 0 \\
\hline Hypoalbuminemia & $8(36.4)$ & 0 & $8(47.1)$ & 0 \\
\hline Anorexia & $7(31.8)$ & 0 & $6(35.3)$ & 0 \\
\hline Bilirubin increased & $7(31.8)$ & $3(13.6)$ & $7(41.2)$ & $3(17.6)$ \\
\hline Diarrhea & $6(27.3)$ & $1(4.5)$ & $4(23.5)$ & $1(5.9)$ \\
\hline Hypothyroidism & $5(22.7)$ & 0 & $4(23.5)$ & 0 \\
\hline Anemia & $5(22.7)$ & 0 & $5(29.4)$ & 0 \\
\hline Thrombocytopenia & $5(22.7)$ & $2(9.1)$ & $5(29.4)$ & $2(11.8)$ \\
\hline Serum amylase increased & $4(18.2)$ & 0 & $3(17.6)$ & 0 \\
\hline Weight loss & $3(13.6)$ & 0 & $3(17.6)$ & 0 \\
\hline
\end{tabular}

HCC, hepatocellular carcinoma.

studies of other second-line MTAs, such as regorafenib, ramucirumab, and cabozantinib $[15,16]$. Although the majority of the patients in those 3 trials had experienced failure of sorafenib treatment and thus were not directly comparable to our patient population, the outcomes of patients in our study who were treated with sorafenib after failure of lenvatinib were not satisfactory. On the other hand, PFS, ORR, and DCR might be more clinically acceptable in patients treated with regorafenib after lenvatinib than in patients treated with sorafenib after lenvatinib. Based on the preclinical study, lenvatinib is known to have a stronger effect on the inhibition of VEGFR and fibroblast growth factor receptor than sorafenib and regorafenib [30]. Meanwhile, sorafenib and regorafenib block PDGFR and KIT strongly as compared with lenvatinib, and sorafenib and regorafenib, unlike lenvatinib, have an effect on RAS/RAF inhibition. Moreover, regorafenib has a distinct molecular target profile and has more potent pharmacological activity than sorafenib $[31,32]$. These differences may be related to the clinical outcomes following lenvatinib therapy. At the present time, regorafenib is generally used for patients with advanced HCC after sorafenib in accordance with the eligibility criteria of the RESORCE trial. That is, regorafenib is indicated for patients with advanced HCC in whom sorafenib has failed and who have confirmed radiological progression during sorafenib treatment and are tolerant of sorafenib. Several studies found that only about one-third of sorafenib-treated patients with advanced HCC were eligible for regorafenib [33-35]. Sequential therapy from sorafenib to regorafenib is an effective treatment stream in both clinical trials and real-world practice, when sorafenib is the first-line agent in patients with advanced HCC $[36,37]$. Recently, Yoo et al. suggested that regorafenib has potential to be effective in patients with advanced HCC regardless of previous treatments [38]. Taken together with our findings, it may be time to abandon the concept that regorafenib should follow sorafenib in patients with failure of lenvatinib treatment. Further prospective clinical trials that investigate the safety and efficacy of regorafenib administered directly after lenvatinib are urgently required.

Regarding adverse events with both sorafenib and regorafenib after lenvatinib, proteinuria was notable (sorafenib: $37.7 \%$, regorafenib: $54.6 \%$ ). The incidence of proteinuria in the present cohort was markedly higher than in both clinical trials and real-world practice $[4,5$, $13,36,39]$. This might be related to the continuation of MTAs after lenvatinib, which are associated with a high incidence of proteinuria. Although the present cohort did not include a large number of patients, further validation of the safety of sequential treatment with MTAs in advanced HCC is necessary.

The results of IMbrave 150, a global phase III study that found that atezolizumab plus bevacizumab significantly prolonged OS compared with sorafenib in patients 
with advanced HCC, have led to a major transition in the treatment of advanced HCC [21]. The standard first-line treatment of advanced HCC has been changing to atezolizumab plus bevacizumab. In other words, treatment is changing rapidly from "the era of multi-MTAs" to "the era of combination immune therapies." Although the prognosis of advanced HCC will be much better, patients who have been administered atezolizumab plus bevacizumab will eventually become refractory to treatment. Since it is expected that MTAs will be used for treatment after atezolizumab plus bevacizumab, lenvatinib will continue to be the first-choice MTA agent in patients with advanced HCC. To improve the clinical outcomes of patients with advanced HCC, establishment of the appropriate treatment stream is urgently required.

The present study had several limitations. First, the study collected data retrospectively, and all treatment decisions were made by the treating physicians. Moreover, because the patients in this cohort were all Japanese and were treated under the Japanese social health-care insurance system, not all results may be replicated in other parts of the world. Global observation studies are needed to verify the safety and efficacy of a wide variety of sequential treatments in patients with advanced HCC, in an era where multiple agents can be used in various combinations as a result of rapid drug development.

In conclusion, sorafenib may not be a candidate for use as a posttreatment agent after lenvatinib, according to the results of the present study. Regorafenib has the potential to become an appropriate posttreatment agent after lenvatinib. Further clinical trials are required to investigate sequential therapy from lenvatinib to regorafenib in patients with advanced HCC. Two other MTAs, ramucirumab and cabozantinib, should also be tested as posttreatment agents after lenvatinib.

\section{Acknowledgement}

The authors would like to thank Enago (www.enago.jp) for the English language review.

\section{Statement of Ethics}

All procedures performed in studies involving human participants were in accordance with the ethical standards of the institutional and/or national research committee. And this research was conducted ethically in accordance with the World Medical Association Declaration of Helsinki. For this type of study, formal consent was not required.

Posttreatment after Lenvatinib

\section{Conflict of Interest Statement}

Sadahisa Ogasawara received grant support from Eisai, Bayer, and Eli Lilly, advisory fees from Eisai, Bayer, MSD, AstraZeneca, and Eli Lilly, and honoraria from Eisai, Bayer, MSD, AstraZeneca, and Eli Lilly. Yoshihiko Ooka received honoraria from Eisai. Naoya Kato received grant support from Eisai, Bayer, Takeda, and Eli Lilly, advisory fees from Eisai, Bayer, and Eli Lilly, and honoraria from Eisai, Bayer, and Eli Lilly. The other authors have no conflicts of interest to declare.

\section{Funding Sources}

This research received no external funding.

\section{Author Contributions}

Conceptualization: Sadahisa Ogasawara; methodology: Sadahisa Ogasawara; formal analysis: Keisuke Koroki, Naoya, Kanogawa, and Sadahisa Ogasawara; investigation: Keisuke Koroki, Naoya Kanogawa, Susumu Maruta, Sadahisa Ogasawara, Yotaro Iino, Masamichi Obu, Tomomi Okubo, Norio Itokawa, Takahiro Maeda, Masanori Inoue, Yuki Haga, Atsuyoshi Seki, Shinichiro Okabe, Yoshihiro Koma, Ryosaku Azemoto, Masanori Atsukawa, Ei Itobayashi, Kenji Ito, Nobuyuki Sugiura, Hideaki Mizumoto, Hidemi Unozawa, Terunao Iwanaga, Takafumi Sakuma, Naoto Fujita, Hiroaki Kanzaki, Kazufumi Kobayashi, Soichiro Kiyono, Masato Nakamura, Tomoko Saito, Takayuki Kondo, Eiichiro Suzuki, Yoshihiko Ooka, Shingo Nakamoto, Akinobu Tawada, and Tetsuhiro Chiba; data curation: Keisuke Koroki, Naoya Kanogawa, and Susumu Maruta; writing - original draft preparation: Keisuke Koroki and Naoya Kanogawa; writing - review and editing: Sadahisa Ogasawara; supervision: Makoto Arai, Tatsuo Kanda, Hitoshi Maruyama, Jun Kato, and Naoya Kato; project administration: Naoya Kato.

\section{Clinical Trial Number}

The Chiba University Research Ethics Committee approved this study (No. 2896).

\section{References}

1 Torre LA, Bray F, Siegel RL, Ferlay J, LortetTieulent J, Jemal A. Global cancer statistics, 2012. CA Cancer J Clin. 2012 Mar;65(2):87-108.

2 Llovet JM, Zucman-Rossi J, Pikarsky E, Sangro B, Schwartz M, Sherman M, et al. Hepatocellular carcinoma. Nat Rev Dis Primers. 2016 Apr;2(2):16018.

3 Llovet JM, Di Bisceglie AM, Bruix J, Kramer BS, Lencioni R, Zhu AX, et al. Design and endpoints of clinical trials in hepatocellular carcinoma. J Natl Cancer Inst. 2008 May 21; 100(10):698-711.

4 Llovet JM, Ricci S, Mazzaferro V, Hilgard P, Gane E, Blanc JF, et al. Sorafenib in advanced hepatocellular carcinoma. N Engl J Med. 2008 Jul 24;359(4):378-90. 
5 Cheng AL, Kang YK, Chen Z, Tsao CJ, Qin S, Kim JS, et al. Efficacy and safety of sorafenib in patients in the Asia-Pacific region with advanced hepatocellular carcinoma: a phase III randomised, double-blind, placebo-controlled trial. Lancet Oncol. 2009 Jan;10(1):25-34.

6 Forner A, Llovet JM, Bruix J. Hepatocellular carcinoma. Lancet. 2012 Mar;379(9822): 1245-55.

7 Johnson PJ, Qin S, Park JW, Poon RT, Raoul JL, Philip PA, et al. Brivanib versus sorafenib as first-line therapy in patients with unresectable, advanced hepatocellular carcinoma: results from the randomized phase III BRISK-FL study. J Clin Oncol. 2013 Oct 1;31(28):3517-24.

8 Cainap C, Qin S, Huang WT, Chung IJ, Pan $\mathrm{H}$, Cheng $\mathrm{Y}$, et al. Linifanib versus sorafenib in patients with advanced hepatocellular carcinoma: results of a randomized phase III trial. J Clin Oncol. 2015 Jan 10;33(2):172-9.

9 Llovet JM, Decaens T, Raoul JL, Boucher E, Kudo M, Chang C, et al. Brivanib in patients with advanced hepatocellular carcinoma who were intolerant to sorafenib or for whom sorafenib failed: results from the randomized phase III BRISK-PS study. J Clin Oncol. 2013 Oct 1;31(28):3509-16.

10 Zhu AX, Park JO, Ryoo BY, Yen CJ, Poon R, Pastorelli D, et al. Ramucirumab versus placebo as second-line treatment in patients with advanced hepatocellular carcinoma following first-line therapy with sorafenib (REACH): a randomised, double-blind, multicentre, phase 3 trial. Lancet Oncol. 2015 Jul;16(7):859-70.

11 Zhu AX, Kudo M, Assenat E, Cattan S, Kang YK, Lim HY, et al. Effect of everolimus on survival in advanced hepatocellular carcinoma after failure of sorafenib: the EVOLVE-1 randomized clinical trial. JAMA. 2014 Jul 2;312(1):57-67.

12 Kudo M, Moriguchi M, Numata K, Hidaka $H$, Tanaka $\mathrm{H}$, Ikeda $\mathrm{M}$, et al. S-1 versus placebo in patients with sorafenib-refractory advanced hepatocellular carcinoma (S-CUBE): a randomised, double-blind, multicentre, phase 3 trial. Lancet Gastroenterol Hepatol. 2017 Jun;2(6):407-17.

13 Bruix J, Qin S, Merle P, Granito A, Huang YH, Bodoky G, et al. Regorafenib for patients with hepatocellular carcinoma who progressed on sorafenib treatment (RESORCE): a randomised, double-blind, placebo-controlled, phase 3 trial. Lancet. 2017 Jan 7;389(10064):56-66.

14 Kudo M, Finn RS, Qin S, Han KH, Ikeda K, Piscaglia F, et al. Lenvatinib versus sorafenib in first-line treatment of patients with unresectable hepatocellular carcinoma: a randomised phase 3 non-inferiority trial. Lancet. 2018 Mar 24;391(10126):1163-73.

15 Abou-Alfa GK, Meyer T, Cheng AL, ElKhoueiry AB, Rimassa L, Ryoo BY, et al. Cabozantinib in patients with advanced and progressing hepatocellular carcinoma. $\mathrm{N}$ Engl J Med. 2018 Jul 5;379(1):54-63.

16 Zhu AX, Kang YK, Yen CJ, Finn RS, Galle PR, Llover JM, et al. Ramucirumab after sorafenib in patients with advanced hepatocellular carcinoma and increased $\alpha$-fetoprotein concentrations (REACH-2): a randomised, double-blind, placebo-controlled, phase 3 trial. Lancet Oncol. 2019 Feb;20(2):282-96.

17 Ikeda K, Kudo M, Kawazoe S, Osaki Y, Ikeda M, Okusaka T, et al. Phase 2 study of lenvatinib in patients with advanced hepatocellular carcinoma. J Gastroenterol. 2017 Apr;52(4):512-9.

18 Hiraoka A, Kumada T, Kariyama K, Takaguchi K, Atsukawa M, Itobayashi E, et al. Clinical features of lenvatinib for unresectable hepatocellular carcinoma in real-world conditions: multicenter analysis. Cancer Med. 2019 Jan;8(1):137-46.

19 Maruta S, Ogasawara S, Ooka Y, Obu M, Inoue $\mathrm{M}$, Itokawa $\mathrm{N}$, et al. Potential of lenvatinib for an expanded indication from the REFLECT trial in patients with advanced hepatocellular carcinoma. Liver Cancer. 2020 Aug; 9(4):382-96.

20 Cheon J, Chon HJ, Bang Y, Park NH, Shin JW, Kim KM, et al. Real-world efficacy and safety of lenvatinib in korean patients with advanced hepatocellular carcinoma: a multicenter retrospective analysis. Liver Cancer. 2020 Sep;9(5):613-24.

21 Finn RS, Qin S, Ikeda M, Galle PR, Ducreux M, Kim TY, et al. Atezolizumab plus bevacizumab in unresectable hepatocellular carcinoma. N Engl J Med. 2020 May 14;382(20):1894-905.

22 Lencioni R, Llovet JM. Modified RECIST (mRECIST) assessment for hepatocellular carcinoma. Semin Liver Dis. 2010 Feb;30(1):52-60.

23 Alsina A, Kudo M, Vogel A, Cheng AL, Tak WY, Ryoo BY, et al. Effects of subsequent systemic anticancer medication following firstline lenvatinib: a post hoc responder analysis from the phase 3 REFLECT study in unresectable hepatocellular carcinoma. Liver Cancer. 2020 Jan;9(1):93-104.

24 Hiraoka A, Kumada T, Fukunishi S, Atsukawa M, Hirooka M, Tsuji K, et al. Post-progression treatment eligibility of unresectable hepatocellular carcinoma patients treated with lenvatinib. Liver Cancer. 2020 Jan;9(1):73-83.

25 Tovoli F, Ielasi L, Casadei-Gardini A, Granito A, Foschi FG, Rovesti G, et al. Management of adverse events with tailored sorafenib dosing prolongs survival of hepatocellular carcinoma patients. J Hepatol. 2019 Dec;71(6):1175-83.

26 Kirino S, Tsuchiya K, Kurosaki M, Kaneko S, Inada $\mathrm{K}$, Yamashita $\mathrm{K}$, et al. Relative dose intensity over the first four weeks of lenvatinib therapy is a factor of favorable response and overall survival in patients with unresectable hepatocellular carcinoma. PLoS One. 2020 Apr 20;15(4):e0231828.

27 Iwamoto H, Suzuki H, Shimose S, Niizeki T, Nakano M, Shirono T, et al. Weekends-off lenvatinib for unresectable hepatocellular carcinoma improves therapeutic response and tolerability toward adverse events. Cancers. 2020 Apr 19;12(4):1010.

28 Shimose S, Iwamoto $\mathrm{H}$, Niizeki $\mathrm{T}$, Shirono $\mathrm{T}$, Noda Y, Kamachi N, et al. Clinical significance of adverse events for patients with unresectable hepatocellular carcinoma treated with lenvatinib: a multicenter retrospective study. Cancers. 2020 Jul 11;12(7):1867.

29 Kudo M. Objective response by mRECIST is an independent prognostic factor of overall survival in systemic therapy for hepatocellular carcinoma. Liver Cancer. 2019 Mar;8(2):73-7.

30 Tian Z, Niu X, Yao W. Receptor tyrosine kinases in osteosarcoma treatment: which is the key target?. Front Oncol. 2020 Aug 28; 10: 1642.

31 Wilhelm SM, Dumas J, Adnane L, Lynch M, Carter CA, Schütz G, et al. Regorafenib (BAY 73-4506): a new oral multikinase inhibitor of angiogenic, stromal and oncogenic receptor tyrosine kinases with potent preclinical antitumor activity. Int J Cancer. 2011 Jul 1;129(1):245-55.

32 Duffaud F, Mir O, Boudou-Rouquette P Piperno-Neumann S, Penel N, Bompas E, et al. Efficacy and safety of regorafenib in adult patients with metastatic osteosarcoma: a noncomparative, randomised, double- blind, placebo-controlled, phase 2 study. Lancet Oncol. 2019 Jan;20(1):120-33.

33 Ogasawara S, Chiba T, Ooka Y, Suzuki E, Maeda T, Yokoyama M, et al. Characteristics of patients with sorafenib-treated advanced hepatocellular carcinoma eligible for secondline treatment. Invest New Drugs. 2018 Apr; 36(2):332-9.

34 Uchikawa S, Kawaoka T, Aikata H, Kodama $\mathrm{K}$, Nishida Y, Inagaki Y, et al. Clinical outcomes of sorafenib treatment failure for advanced hepatocellular carcinoma and candidates for regorafenib treatment in real-world practice. Hepatol Res. 2018 Sep;48(10):81420.

35 Terashima T, Yamashita T, Sunagozaka H, Arai K, Kawaguchi K, Kitamura K, et al. Analysis of the liver functional reserve of patients with advanced hepatocellular carcinoma undergoing sorafenib treatment: Prospects for regorafenib therapy. Hepatol Res. 2018 Nov; 48(12):956-66.

36 Ogasawara S, Ooka Y, Itokawa N, Inoue M, Okabe S, Seki A, et al. Sequential therapy with sorafenib and regorafenib for advanced hepatocellular carcinoma: a multicenter retrospective study in Japan. Invest New Drugs. 2020 Feb;38(1):172-80.

37 Wang W, Tsuchiya K, Kurosaki M, Yasui Y, Inada $\mathrm{K}$, Kirino $\mathrm{S}$, et al. Sorafenib-regorafenib sequential therapy in Japanese patients with unresectable hepatocellular carcinoma-relative dose intensity and post-regorafenib therapies in real world practice. Cancers. 2019 Oct;11(10): 1517.

38 Yoo C, Byeon S, Bang Y, Cheon J, Kim JW, $\mathrm{Kim} \mathrm{JH}$, et al. Regorafenib in previously treated advanced hepatocellular carcinoma: impact of prior immunotherapy and adverse events. Liver Int. 2020 Sep;40(9):2263-71.

39 Ogasawara S, Chiba T, Ooka Y, Kanogawa N, Saito T, Motoyama T, et al. Sorafenib treatment in Child-Pugh A and B patients with advanced hepatocellular carcinoma: safety, efficacy and prognostic factors. Invest New Drugs. 2015 Jun;33(3):729-39. 